\title{
DERMATOLOGIC THERAPY
}

\section{Methylene blue vs. methyl aminolevulinate photodynamic therapy in the treatment of mild-to-moderate toenail onychomycosis: short and medium-term effects.}

\begin{tabular}{|c|c|}
\hline Journal: & Dermatologic Therapy \\
\hline Manuscript ID & DTH-20-1525.R1 \\
\hline Wiley - Manuscript type: & Original Article \\
\hline Keywords: & $\begin{array}{l}\text { Infection- bacterial/Fungal/Viral, Nail disorders, Photodynamic therapy, } \\
\text { Methylene blue, Methyl aminolevulinate }\end{array}$ \\
\hline Abstract: & $\begin{array}{l}\text { Photodynamic therapy (PDT) has emerged as an interesting alternative } \\
\text { option for onychomycosis treatment. The impact of a specific } \\
\text { photosensitizer (PS) on the final result is an important factor to consider. } \\
\text { We conducted a short- and medium-term controlled trial to compare the } \\
\text { effectiveness of PDT in the treatment of mild-to-moderate } \\
\text { onychomycosis when it is mediated by two different PSs. Twenty patients } \\
\text { were randomized to receive } 9 \text { sessions of PDT distributed over } 16 \text { weeks } \\
\text { mediated either by methylene blue (MB/PDT group) or methyl } \\
\text { aminolevulinate (MAL/PDT group). Onychomycosis severity index (OSI) } \\
\text { and nail involvement were checked along the study. Complete cure, } \\
\text { treatment success and clinical improvement were tabulated at } 16 \text { and } \\
40-\text { week follow-ups. OSI scores decreased significantly along the study, } \\
\text { from } 12.1 \pm 5.4 \text { to } 3.6 \pm 3.2 \text { (MB/PDT group) and from } 14.8 \pm 6.0 \text { to } \\
5.4 \pm 4.4 \text { (MAL/PDT group). At } 16 \text {-week follow-up, only } 20 \% \text { of the } \\
\text { patients in the MB/PDT group reached complete cure and none in the } \\
\text { group of MAL/PDT. At } 40 \text {-week follow-up, complete cure rates were } 70 \% \\
\text { and } 40 \% \text { in the MB/PDT group and MAL/PDT group respectively. Both } \\
\text { modalities showed good outcomes in treatment of moderate toenail } \\
\text { onychomycosis. MB/PDT showed a faster action but with relapse rates } \\
\text { slightly higher than MAL/PDT. }\end{array}$ \\
\hline
\end{tabular}

\section{SCHOLARONE Manuscripts}


Title: Methylene blue vs. methyl aminolevulinate photodynamic therapy in the treatment of mild-to-moderate toenail onychomycosis: short and medium-term effects.

Running head: MB vs. MAL in mild-to-moderate onychomycosis.

Authors: Enrique Alberdi ${ }^{1}$, Clara Gómez ${ }^{*}$

1 Private clinic of Dr. Alberdi, Aviador Zorita 25, 28020 Madrid

2 Institute of Physical Chemistry Rocasolano, CSIC, Serrano 119, 28006 Madrid, Spain

\author{
Address of authors: \\ Clara Gómez (author for correspondence)*: \\ Institute of Physical Chemistry Rocasolano \\ Spanish National Research Council, CSIC \\ C/ Serrano 119, 28006 Madrid \\ Tel.: +34-91-7459532 \\ Fax.: +34-91-5642431 \\ E-mail address: c.gomez@iqfr.csic.es
}

\title{
Acknowledgements
}

This research was supported by a grant from the Eugenio Rodríguez Pascual Foundation

(Madrid, Spain) and by the Spanish Research Project MICINN (Ref.: MAT 201783856-C3). Authors wish to thank Professor $\mathrm{M}^{\mathrm{a}}$ José Ortiz for her assistance with the ethical evaluation of the study and Dr. Santiago Cano for his assistance with the statistical analysis. 


\begin{abstract}
Photodynamic therapy (PDT) has emerged as an interesting alternative option for onychomycosis treatment. The impact of a specific photosensitizer (PS) on the final result is an important factor to consider. We conducted a short- and medium-term controlled trial to compare the effectiveness of PDT in the treatment of mild-tomoderate onychomycosis when it is mediated by two different PSs. Twenty patients were randomized to receive 9 sessions of PDT distributed over 16 weeks mediated either by methylene blue (MB/PDT group) or methyl aminolevulinate (MAL/PDT group). Onychomycosis severity index (OSI) and nail involvement were checked along the study. Complete cure, treatment success and clinical improvement were tabulated at 16 and 40-week follow-ups. OSI scores decreased significantly along the study, from $12.1 \pm 5.4$ to $3.6 \pm 3.2$ (MB/PDT group) and from $14.8 \pm 6.0$ to $5.4 \pm 4.4$ (MAL/PDT group). At 16 -week follow-up, only $20 \%$ of the patients in the MB/PDT group reached complete cure and none in the group of MAL/PDT. At 40-week follow-up, complete cure rates were $70 \%$ and $40 \%$ in the $\mathrm{MB} / \mathrm{PDT}$ group and MAL/PDT group respectively. Both modalities showed good outcomes in treatment of moderate toenail onychomycosis. MB/PDT showed a faster action but with relapse rates slightly higher than MAL/PDT.
\end{abstract}

\title{
Keywords:
}

Mild-to-moderate onychomycosis; photodynamic therapy; methylene blue; methyl aminolevulinate. 


\section{INTRODUCCION}

Cutaneous fungal infections are one of the most prevalent skin problems worldwide. Many species of fungi can cause skin infections. Onychomycosis is the most prevalent onychopathy that comprises $50 \%$ of nail disorders worldwide. ${ }^{1}$ In the USA, $90 \%$ of the fungi causing onychomycosis are dermatophytes or yeast such as Trichophyton rubrum (71\%), Trichophyton interdigitale (20\%) and Candida albicans $(5.6 \%) .{ }^{1}$ T. rubrum is a representative anthropophilic strain that causes human skin infections including tinea pedis and onychomycosis. T. interdigitale is another well-known dermatophytic fungus, frequently observed along with T. rubrum. ${ }^{2}$ Longitudinal striae, "jagged edge" with spikes (pattern results from distal-to-proximal invasion of dermatophytes) and "ruin appearance" are the three major onychoscopic features associated with onychomicosis. ${ }^{3}$ Patients with onychomycosis more frequently showed concomitant tinea pedis and show a clear worsening of nail changes in the last years. ${ }^{4}$

The severity and percentage of nail involvement are usually considered the main prognostic factors for the treatment of onychomycosis. ${ }^{5}$ In most countries, if there is less than $50 \%$ involvement of diseased nail plate and less than three nails involved, topical therapy is an appropriate consideration. ${ }^{6}$ Topical treatments should also be used for treatment of white superficial onychomycosis. In these cases, among the innovative physical approaches for moderate onychomycoses are nail peeling, ${ }^{7}$ thermal disruption of fungal biofilm by lasers and photochemical damage by photodynamic therapy (PDT). ${ }^{8}$ Lasers can elicit a fungicidal effect by photothermally heating the fungal mycelium through selective photothermolysis. ${ }^{9,10}$ PDT is a noninvasive therapy that requires light activation of a photosensitizer (PS) in the presence of oxygen which 
generates reactive oxygen species leading to selective and highly localized destruction of "abnormal" cells by necrosis or apoptosis. Fungi are able to absorb photosensitizers so PDT can be an alternative way of treating onychomycosis. A few clinical trials have been published on PDT, demonstrating that PDT could be a promising alternative to conventional antifungal therapy. ${ }^{11,12}$

We conducted a short- and medium-term controlled trial to evaluate the effectiveness of PDT in the treatment of mild-to-moderate onychomycosis. In addition, a comparison between methylene blue (MB) and methyl aminolevulinate (MAL) as PSs was carried out in terms of clinical and mycological cure.

\section{PATIENTS AND METHODS}

A controlled trial of 40 -week duration was designed. Patients with mild-moderate signs of distal and lateral subungual onychomycosis (DLSO) in the first toe, (infection confirmed by PAS stain and microbiological culture to identify only dermatophytes as causative agents), were randomly allocated into two different treatment groups by means of a computer-generated random list: group 1: MB/PDT or group 2: MAL/ PDT (Figure 1). Characteristics of the recruited patients or inclusion criteria are shown in Table 1. Inclusion criteria: OSI $\sim 13 ; \sim 35 \%$ nail involvement. Exclusion criteria were as follows: less than 18 years of age, glucose-6-phosphate dehydrogenase deficiency (which contraindicates the use of MB), porphyria diagnosed (which contraindicates the use of MAL), suffering from any disease that can cause nail abnormalities and can interfere with evaluation (e.g. tinea pedis, psoriasis or lichen planus), internal diseases, use of topical or systemic antifungals for at least 6 months before enrollment in the trial and pregnancy (Figure 1). 
A local Ethics Committee approved the study (Hospital Clínico San Carlos in Madrid, Spain; Internal code: 17/501-E) in accordance with the Declaration of Helsinki, the principles of Good Clinical Practice and all applicable regulatory requirements. All subjects provided written informed consent form before their inclusion in the study.

Two weeks before the experimental assessment of treatment started, subjects received instruction of the pretreatment with $40 \%$ urea to be performed regularly during research monitoring and 3-7 days before a PDT session. The treatment consisted in nine PDT sessions distributed over 16 weeks. In parallel, clinical photographs, microbiological samples for fungal cultures and nail samples for histology (PAS stain) were taken at different time-points in order to assess the efficacy of each treatment (Figure 1).

\subsection{Interventions}

Plates of the affected nails were softened with $40 \%$ urea ointment. Periungual skin was protected with vaseline to avoid any lesion produced by the urea. Upon application of the urea, the nail was covered with occlusive dressing for 12-24 h. In order not to excessively macerate the periungual skin the daily softening treatment with urea was limited to 3 days in toenails with hyperkeratosis less than $2 \mathrm{~mm}$, whereas it was maintained during 7 days in toenails with hyperkeratosis greater than $2 \mathrm{~mm}$.

\section{Photodynamic therapy}

MB/PDT protocol: In each PDT session, MB (at a concentration of $2 \%$ in aqueous solution) was applied to the nail and surrounding area, leaving the solution in contact with the nail for $3 \mathrm{~min}$. 
$\underline{M A L / P D T \text { protocol: }}$ In each PDT session, MAL (Metvix ${ }^{\circledR}$, Galderma, La Defense Cedex, France) was applied to the nail plate under occlusion and protected from light for $3 h$.

In both groups, after incubation period, irradiation was carried out with a $635 \mathrm{~nm}$ LEDs lamp (Aktilite ${ }^{\circledR}$ CL128, Photocure ASA, Oslo, Norway) using a fluence of $37 \mathrm{~J} / \mathrm{cm}^{2}$. The lamp was placed at $10 \mathrm{~cm}$ from the skin and the illumination time was calculated automatically (10 min.). The same protocol was repeated nine times at two-week intervals. Side-effects were recorded during each session of PDT.

\subsection{Outcomes}

Clinical clearance was assessed by a dermatologist by scoring the photographs taken over the study by using the onychomycosis severity index (OSI) (mild $\leq 5$, moderate: 615 and severe: 16-35) and percentage of nail involvement. Complete clinical clearance was defined by a completely normal appearance of the nail or the presence of $\leq 5 \%$ nail plate involvement in onychomycosis. Mycological clearance was assessed after the results obtained after fungal culture and by biopsy of the nail affected by PAS stain (Figure 2). The biopsy was carried out by cutting a fragment of the distal portion of the nail plate at least $3 \mathrm{~mm}$ wide together with the attached subungueal keratosis. The extracted samples were then stained with periodic acid Schiff (PAS) and their subsequent histological study allowed detecting the presence of fungi in the lower portion of the nail plate (Figure 2). Complete cure was defined as the addition of clinical and mycological cure.

The difference between baseline OSI score and those obtained at 16 and 40-week follow-up was transformed in percentage of change. Values obtained were classified as 
follows: i) $0-25 \%$ (no improvement), ii) $26-50 \%$ (mild improvement), iii) $51-75 \%$ (moderate improvement) and iv) $76-100 \%$ (outstanding improvement). ${ }^{13}$

As reported by Shemer et al., ${ }^{14}$ treatment success and clinical improvement can also be tabulated to achieve a more complete analysis of the results. In this way, treatment success was defined as mycological cure + less than $10 \%$ of nail affected. Clinical improvement was defined as mycological cure + less than $25 \%$ of nail affected.

In order to assess the degree of satisfaction of the patient with the applied treatment, in each PDT session, the patient was asked whether they noticed pain or discomfort in the session itself or during the time elapsed between sessions.

\subsection{Sample size}

As a change of 10 points or more in OSI scores is considered significant, ${ }^{15}$ by using IBM SPSS Sample Power 3.0 software it was estimated that a sample size of 9 patients per group was required to detect a 10-point change in OSI scores with $90 \%$ power, $\alpha=0.05$ and standard deviation of 6 . A total sample of 24 patients with only one toenail affected by DLSO (12 per group) were randomly allocated anticipating in this way possible dropouts (Figure 1).

\subsection{Statistical analysis}

Statistical analysis was performed with a SPSS software package for Windows (version 25.0; SPSS, Chicago, III). Results were expressed as arithmetic mean and standard deviation. The Shappiro-Wilk test was used to verify the normality of distribution of quantitative variables, and them they were analyzed by bifactorial repeated measures ANOVA test (considering an intra-subject factor (time) and an inter-subject factor 
(treatment)) with the post hoc Bonferroni correction for identification of intra-group differences. Significance of the obtained results ( $p$ value) was judged at the 5\% level.

\section{RESULTS}

After the application of restrictive inclusion and exclusion criteria and taking into account dropouts and exclusion of patients with incompletely recorded histories, the data of final 20 subjects (10 patients Group 1: MB/PDT; 10 patients Group 2: MAL/PDT) were analyzed (Figure 1). The groups were divided obtaining similar distributions of OSI and percentage of disease involvement (Table 1). Baseline clinical and laboratory data of included patients are summarized in Table 1.

Complications or side effects were not observed during or after application of either PDT modality. In the case of MAL/PDT, some patients referred pain or itching sensation, but only during irradiation time. $\mathrm{MB}$ application resulted in temporary discoloration of the nail. In any case, the level of patient satisfaction was high.

Figure 3 and Table 2 show the evolution of the clinical clearance during and after treatment. A progressive reduction in the OSI values was observed after treatment. Intragroup significant differences were observed in both treatment modalities at 16- and 40-week follow-up from baseline. Intergroup significant differences attending OSI values were not observed. Regarding the degree of improvement, significant differences between 16-week follow-up and 40-week follow-up were detected with MAL/PDT, while after MB/PDT the degree of improvement at 16- and 40-week follow-up was similar. Significant differences between groups at 16-week follow-up were observed, indicating that the healing process is significantly faster with MB/PDT. Finally, the degree of disease involvement follows a trend similar to OSI scores, significant 
intragroup differences at 16- and 40-week follow-ups from baseline and between 16and 40-week follow-up in both treatment modalities and no point of significant differences between them.

Evolution of the mycological clearance is also shown in Table 2. Complete mycological cure rates were $70 \%$ and $60 \%$ at 40 -week follow-up in MB/PDT and MAL/PDT groups respectively. The fraction of patients who reached mycological cure at 40 -week followup was significant in both groups respect to baseline and 16-week follow-up. The evolution in mycological cure showed no significant intergroup difference. All MB/PDT patients that reached mycological cure reached complete cure as well (Table 3). Among MAL/PDT patients that reached mycological cure, $67 \%$ reached complete cure, $17 \%$ reached treatment success and 17\% reached clinical improvement (Table 3). It is important to point out that during the time interval studied two MB/PDT patients suffered relapses despite the faster healing rate.

\section{DISCUSION}

Onychomycosis is a chronic, hard-to-treat infection of the nails that can have a significant impact on patients' quality of life. Therapeutic guidelines for treatment do exist, ${ }^{16}$ but they are not yet up to date on the latest scientific knowledge. ${ }^{5}$ Novel therapies based on light have been explored as a possible and good alternative treatment for onychomycosis. Selective destruction of fungal structures seems to be the mechanism of action of these novel therapies. ${ }^{17}$ PDT has been shown to be effective on photoinactivation of multiple species of fungi including the most common cause of onychomycosis, T. rubrum. ${ }^{18}$ Moreover, PDT does not induce systemic side effects and has the advantage of a low probability of selection of resistant microorganism, since the resistance to ROS is virtually impossible. ${ }^{19}$ 
Recently a review study concludes that topical therapy for toenail onychomycosis produces clinical and mycological cure but only in patients with mild to moderate toenail onychomycosis $(<50 \%$ nail involvement $) .{ }^{20}$ We propose PDT treatment as an alternative to topical treatment in these cases with mild-moderate disease involvement. For this reason, good candidates for receiving these PDT modalities are patients with superficial onychomycosis or DLSO that involves less than $50 \%$ of the nail plate surface area (without matrix involvement and a nail plate thickness less than $2 \mathrm{~mm}$ ). This study evaluated the efficacy and safety of MB/PDT vs. MAL/PDT. At 40 week follow-up, complete cure rates were $70 \%$ and $40 \%$ in the MB/PDT group and MAL/PDT group respectively. These complete cure rates are higher than those obtained with topical agents on toenail DLSO with $20-50 \%$ clinical involvement. Compare for example the $17.8 \%$ and $15.2 \%$ of complete cure rates reported at week 52 with efinaconazole $10 \% .{ }^{21}$ In addition, the results here showed present higher cure rates than the $28.6 \%$ of complete cure obtained at week 60 after 48 weeks of treatment with oncedaily $5 \%$ tavaborole solution, ${ }^{22}$ and the $5.8 \%$ of the complete cure at week 60 after treatment with standard $8 \%$ ciclopirox nail lacquer ${ }^{5}$ or the $20 \%$ of negative mycological culture at 9 months following $5 \%$ amorolfine nail lacquer. ${ }^{23}$

Urea pretreatment could boost the difference as for the degree of improvement between MB/PDT and MAL/PDT at 16-week follow-up, thus the similar degree of improvement at 16 and 40-week follow-ups for MB/PDT, as urea exerts an emollient/keratolytic action and therefore it allows a better MB/MAL and LED light penetration across the nail plate. ${ }^{15}$ The hydrophilic MB diffuses better that MAL across the nail previously treated with urea and thus its action is faster than that of MAL. Recently, there are more and more studies which propose a previous pretreatment of the affected nail with fractionated $\mathrm{CO}_{2}$ or Er:YAG lasers, enhancing the absorption of PS without the needed 
of the use of urea $40 \%$ and increasing the aesthetic outcome. This combination has as a counterpart a greater sensation of pain. ${ }^{24,25}$

The common protocol for onychomycosis treatment carried out in the studies published using MAL as PS consisted of 3 PDT sessions, separated by an interval of 1 or 2 weeks, using MAL $16 \%, 3 \mathrm{~h}$ as incubation time and red light $\left(\lambda=630 \mathrm{~nm}, 37 \mathrm{~J} / \mathrm{cm}^{2}\right) .{ }^{15,26}$ In contrast, studies using MB consisted of 12 sessions with an interval of 15 days between them, $3 \mathrm{~min}$ as incubation time, and red light $\left(\lambda=630 \mathrm{~nm}, 36-18 \mathrm{~J} / \mathrm{cm}^{2}\right)$. using $\mathrm{MB}$ $(2 \%) \cdot{ }^{27,28}$

In order to unify protocols and for an easier PS comparison, in the present study with patients affected by mild-moderate onychomycosis, nine sessions of PDT were applied, separated by an interval of 2 weeks, using the same light system operating at $\lambda=630 \mathrm{~nm}$, $37 \mathrm{~J} / \mathrm{cm}^{2}$. The final aim was to both ensure total healing and avoid the manifestation of the recurrence. Success results were obtained after the application of a similar protocol (8 PDT/light sessions instead of 9) into a clinical trial with 40 patients randomly assigned to MB/PDT or IPL in the treatment of mild-moderate toenail onychomycosis, showing clinical improvement rates of $70 \%$ and $80 \%$, respectively at 28 weeks from baseline. $^{11}$

The fact that onychomycosis is usually not an immediate threat to their health may lead patients to delay treatment due to the high cost of antifungal medications and their potentially harmful side effects. Patients that have had onychomycosis for shorter periods have generally better cure rates than those who have had the disease for longer periods, hence, the most prudent decision is to initiate a topical therapy as early as possible. ${ }^{29}$ Some of our patients did not experience complete cure at this medium-term period of evaluation of 40 weeks $(30 \%$ in the MB/PDT group and $60 \%$ in the 
MAL/PDT group). Among the factors that could predispose to not obtaining complete cure are slow nail growth, nail abnormalities and poor circulation, all of which may increase with age. ${ }^{30}$ Subungueal dermatophytoma that is more common in older people due to a longer duration of onychomycosis is another predictor of poorer response to therapy. ${ }^{31}$ In the present study the same sequencing of PDT sessions was applied for the two modalities of PS in order to achieve a better comparison between the procedures. Moreover, most of the subjects with similar age were diagnosed with onychomychosis caused only by dermatophytes, which is easier to eradicate by systemic and topical drugs. ${ }^{32}$ In the present study, although both modalities reach similar mycological cure rates at 40-week follow-up, the clinical cure rates were slower after MAL/PDT than after MB/PDT. After randomization process, dropouts (exclusion of patients for not attending sessions/follow-up), some discrepancy regarding the chronicity of the disease was evidenced between both groups. The delay in healing of patients treated with MAL/PDT compared to those treated with MB/PDT may be due to the longer duration of the disease in the former group.

Further studies are needed to define optimal PDT protocol (light source (laser/LED), fluence/radiance, light exposure time, number and frequency of treatments required, optimal PS -optimal concentration and incubation time-, combination with topical or systemic antifungal agents...), to adjust the therapeutic outcome in accordance with the onychomychosis involvement degree.

\section{CONCLUSIONS}

Results of the present clinical trial suggest that PDT mediated by MB or MAL is a safe and effective method with satisfactory results in the treatment of mild-to-moderate toenail onychomycosis. In order to optimize the protocol, further evaluation in larger patient population would be required and in a long-term interval. 


\section{Declaration of interest}

The authors have no other relevant affiliation or financial involvement with any organization or entity with a financial interest in or financial conflict with the subject matter or materials discussed in the manuscript apart from those disclosed.

\section{Authors contributions}

Both authors had the same contribution in conducting the manuscript. Both authors read and approved the manuscript in its submitted form. 


\section{REFERENCES}

[1] Faergemann J, Baran R. Epidemiology, clinical presentation and diagnosis of onychomycosis. Br J Dermatol. 2003;149 Suppl 65: 1-4.

[2] Gräser Y, Scott J, Summerbell R. The new species concept in dermatophytes - a polyphasic approach. Mycopathologia. 2008; 166 (5-6): 239-256.

[3] Nakamura RC, Costa MC. Dermatoscopic findings in the most frequent onychopathies: a descriptive analyses of 500 cases. Int J Dermatol. 2012; 51(4):483485.

[4] Ramos Pinheiro R, Dias Domingues T, Sousa V, Galhardas C, Apetato M, Lencastre A. A comparative study of onychomycosis and traumatic toenail onychodystrophy dermoscopic patters. J Eur Acad Dermatol Venereol. 2019; 33(4):786-792.

[5] Piraccini BM, Tosti A. Ciclopirox hydroxypropyl chitosan: efficacy in mild-tomoderate Onychomycosis. Skin Appendage Disord. 2018; 5(1):13-19.

[6] Gupta AK, Mays RR, Versteeg SG, Piraccini BM, Takwale A, Shemer A, Babaev M, Grover C, Di Chiacchio NG, Taborda PRO, Taborda VBA, Shear NH, Piguet V, Tosti A. Global perspectives for the management of onychomycosis. Int J Dermatol. 2019; 58(19):1118-1129.

[7] Sonthalia S, Jakhar D, Yadav P, Kaur I. Chemical peeling as an innovative treatment alternative to oral antifungals for onychomycosis in special circumstances. Skin Appendage Disord. 2019; 5(3):181-185.

[8] Sonthalia S, Goldust M. Innovative physical approaches for onychomycosis: peeling, lasers, and beyond. Skin Appendage Disord. 2019; 5(4):197-200. 
[9] Altshuler GB, Anderson RR, Manstein D, Zenzie HH, Smirnov MZ. Extended theory of selective photothermolysis. Lasers Surg Med. 2001; 29(5): 416-432.

[10] Anderson RR, Parrish JA. Selective photothermolysis: precise microsurgery by selective absorption of pulsed radiation. Science. 1983; 220(4596): 524-527.

[11] Alberdi E, Gómez C. Efficiency of methylene blue-mediated photodynamic therapy vs intense pulsed light in the treatment of onychomycosis in the toenails. Photodermatol Photoimmunol Photomed. 2019; 35(2): 69-77.

[12] Morgado LF, Trávolo ARF, Muehlmann LA, Narcizo PS, Nunes RB, Pereira PAG, Py-Daniel KR, Jiang CS, Gu J, Azevedo RB, Longo JPF. Photodynamic therapy treatment of onychomycosis with aluminium-phthalocyanine chloride nanoemulsions: a proof of concept clinical trial. J Photochem Photobiol B 2017; 173:266-270.

[13] El-Tatawy RA, Aliweh HA, Hegab DS, Talaat RAZ, Shams Eldeen MA. Fractional carbon dioxide laser and topical tioconazole in the treatment of fingernail onychomycosis. Lasers Med Sci. 2019; 34(9):1873-1880.

[14] Shemer A, Gupta AK, Kamshov S, Babaev M, Hermush V, Farhi R, Daniel CR 3rd, Foley KA. Continuous terbinafine and pulse itraconazole for the treatment of nondermatophyte mold toenail onychomycosis. J Dermatolog Treat. 2019 DOI: 10.1080/09546634.2019.1654598.

[15] Gilaberte Y, Robres MP, Frías MP, García-Doval I, Rezusta A, Aspiroz C. Methylaminolevulinate photodynamic therapy for onychomycosis: a multicentre, randomized, controlled clinical trial. J Eur Acad Dermatol Venereol. 2017; 31(2):347354. 
[16] Elewski BE. Onichomycosis. Treatment, quality of life, an economic issues. Am J Clin Dermatol. 2000; 1(1):19-26.

[17] Zalacain A, Merlos A, Planell E, Cantadori EG, Vinuesa T, Viñas M. Clinical laser treatment of toenail onychomycoses. Lasers Med Sci. 2018; 33(4):927-933.

[18] Piraccini BM, Rech G, Tosti A. Photodynamic therapy of onychomycosis caused by Trichophyton rubrum. J Am Acad Dermatol. 2008; 59 (5Suppl): S75-S76.

[19] da Silva AP, Chiandrone DJ, Tinta JWR, Kurachi C, Inada NM, Bagnato VS. Development and comparison of two devices for treatment of onychomycosis by photodynamic therapy. J Biomed Opt. 2015; 20(6): 061109.

[20] Gupta AK, Daigle D, Foley KA. Topical therapy for toenail onychomycosis: an evidence-based review. Am J Clin Dermatol. 2014; 15(6): 489-502.

[21] Elewski BE, Rich P, Pollak P, Pariser DM, Watanabe S, Senda H, Ieda C, Smith K, Pillai R, Ramakrishna T, Olin JT. Eficonazole 10\% solution in the treatment of toenail onychomycosis: Two phase III multicenter, randomized, double-blind studies. $J$ Am Acad Dermatol. 2013; 68(4): 600-608.

[22] Gupta AK, Hall S, Zane LT, Lipner SR, Rich P. Evaluation of the efficacy and safety of tavaborole topical solution, 5\% in the treatment of onychomycosis of the toenail in adults: a pooled analysis of an 8-week, post-study follow-up from two randomized phase 3 studies. J Dermatolog Treat. 2018; 29(1): 44-48.

[23] Koren A, Salameh F, Sprecher E, Artzi O. Laser-assisted Photodynamic Therapy or Lasser-assisted Amorolfine Lacquer Delivery for Treatment of Toenail Onychomycosis: An Open-label Comparative Study. Acta Derm Venereol. 2018; 98(4): 467-468. 
[24] Zaki AM, Abdo HM, Ebadah MA, Ibrahim SM. Fractional $\mathrm{CO}_{2}$ laser plus topical antifungal versus fractional $\mathrm{CO}_{2}$ laser versus topical antifungal in the treatment of onychomycosis. Dermatol Ther. 2020;33(1): e13155. doi: 10.1111/dth.13155

[25] Zhang J, Lu S, Huang H, Li X, Cai W, Ma J, Xi L. Combination therapy for onychomycosis using a fractional 2940-nm Er:YAG laser and $5 \%$ amorolfine lacquer. Lasers Med Sci. 2016; 31(7):1391-1396.

[26] Gilaberte Y, Aspiroz C, Martes MP, Alcalde V, Espinel-Ingroff A, Rezusta A. Treatment of refractory fingernail onychomycosis caused by nondermatophyte molds with methylaminolevulinate photodynamic therapy. J Am Acad Dermatol. 2011; 65(3): 669-671.

[27] Figueiredo Souza LW, Souza SV, Botelho AC. Randomized controlled trial comparing photodynamic therapy based on methylene blue dye and fluconazole for toenail onychomycosis. Dermatol Ther. 2014; 27(1): 43-47.

[28] Souza LW, Souza SV, Botelho AC. Endonyx toenail onychomycosis caused by Trichophyton rubrum: treatment with photodynamic therapy based on methylene blue dye. An Bras Dermatol. 2013; 88(6), 1019-1021.

[29] Tosti A, Hay R, Arenas-Guzmán R. Patients at risk of onychomycosis-risk factor identification and active prevention. J Eur Acad Dermatol Venereol. 2005; 19 Suppl 1:13-16.

[30] Ko JY, Lee HE, Jae H, et al. Cure rate, duration required for complete cure and recurrence rate of onychomycosis according to clinical factors in Korean patients. Mycoses. 2011; 54(5): e384-388. 
[31] Murdan S. Nail disorders in older people, and aspects of their pharmaceutical treatment. Int J Pharmaceutics 2016; 512: 405-411.

[32] Gupta AK, Drummond-Main C, Cooper EA, Brintnell W, Piraccini BM, Tosti A. Systematic review of nondermatophyte mold onychomycosis: diagnosis, clinical types, epidemiology, and treatment. J Am Acad Dermatol. 2012; 66(3): 494-502. 
Table 1.- Baseline demographics of recruited patients. $(\mathrm{n}=$ number of patients; $(\%)=$ percentage of patients)

\begin{tabular}{|l|l|l|}
\cline { 2 - 3 } \multicolumn{1}{c|}{} & \multicolumn{1}{c|}{ MB/PDT } & \multicolumn{1}{c|}{ MAL/PDT } \\
\hline Sex & $4(40 \%)$ & $9(90 \%)$ \\
Male [n (\%)] & $6(60 \%)$ & $1(10 \%)$ \\
Female [n (\%)] & $63.6 \pm 6.7 ;[53,71] ; 63$ & $64.7 \pm 11.8 ;[43,78] ; 66$ \\
Age [mean $\pm(\mathrm{SD})]$; range; median & $10(100 \%)$ & $10(100 \%)$ \\
\hline $\begin{array}{l}\text { Hystological analysis [n (\%)] } \\
\text { PAS stain + } \\
\text { PAS stain - }\end{array}$ & $0(0 \%)$ & $0(0 \%)$ \\
\hline $\begin{array}{l}\text { Microbiological analysis [n (\%)] } \\
\text { T rubrum } \\
\text { T interdigitale }\end{array}$ & $9(90 \%)$ & $8(80 \%)$ \\
\hline Baseline OSI [mean $\pm(\mathrm{SD})]$ & $1(10 \%)$ & $2(20 \%)$ \\
\hline Chronicity (years) $[\operatorname{mean} \pm(\mathrm{SD})]$ & $12.1 \pm 5.4$ & $14.8 \pm 6.0$ \\
\hline Nail involvement $(\%)[m e a n \pm(\mathrm{SD})]$ & $37.5 \pm 20.4$ & $3.2 \pm 2.8$ \\
\hline
\end{tabular}


Table 2.- Evolution of OSI, percentage of disease involvement and mycological response during the study. $(\mathrm{n}=$ number of patients; $(\%)=$ percentage of patients $)$

\begin{tabular}{|c|c|c|}
\hline & $\mathrm{MB} / \mathrm{PDT}$ & MAL/PDT \\
\hline Baseline OSI [mean $\pm(\mathrm{SD})]$ & $12.1 \pm 5.4$ & $14.8 \pm 6.0$ \\
\hline 16-w follow-up OSI [mean $\pm(\mathrm{SD})]$ & $2.6 \pm 1.9^{a}$ & $9.2 \pm 5.1^{\mathrm{a}}$ \\
\hline 40-w follow-up OSI [mean $\pm(\mathrm{SD})]$ & $3.6 \pm 3.2^{\mathrm{a}}$ & $5.4 \pm 4.4^{\mathrm{a}}$ \\
\hline $\begin{array}{l}\text { Degree of improvement at } 16-\mathrm{w} \text { follow-up [n (\%)] } \\
\text { No }(0-25 \%) \\
\text { Mild }(26-50 \%) \\
\text { Moderate }(51-75 \%) \\
\text { Outstanding }(76-100 \%) \\
\text { Mean percentage of improvement degree }\end{array}$ & $\begin{array}{l}0(0) \\
1(10) \\
5(50) \\
4(40) \\
77.4 \%\end{array}$ & $\begin{array}{l}6(60) \\
1(10) \\
3(30) \\
0(0) \\
36.1 \%\end{array}$ \\
\hline Significance between groups & \multicolumn{2}{|c|}{$p<0.05(0.001)$} \\
\hline $\begin{array}{l}\text { Degree of improvement at } 40-\mathrm{w} \text { follow-up [n (\%)] } \\
\text { No }(0-25 \%) \\
\text { Mild }(26-50 \%) \\
\text { Moderate }(51-75 \%) \\
\text { Outstanding }(76-100 \%) \\
\text { Mean percentage of improvement degree }\end{array}$ & $\begin{array}{l}2(20) \\
0(0) \\
2(20) \\
6(60) \\
76.2 \%\end{array}$ & $\begin{array}{l}1(10) \\
3(30) \\
2(20) \\
4(40) \\
66.1 \% b\end{array}$ \\
\hline Significance between groups & \multicolumn{2}{|c|}{$p>0.05$} \\
\hline Baseline nail involvement (\%) [mean $\pm(\mathrm{SD})]$ & $37.5 \pm 20.4$ & $33.0 \pm 18.4$ \\
\hline 16-w follow-up nail involvement (\%) [mean $\pm(\mathrm{SD})]$ & $11.0 \pm 7.7^{\mathrm{a}}$ & $19.0 \pm 9.7^{\mathrm{a}}$ \\
\hline 40-w follow-up nail involvement $(\%)[$ mean $\pm(S D)]$ & $4.0 \pm 3.6^{\mathbf{a}, \mathbf{b}}$ & $14.5 \pm 12.8^{\mathrm{a}, \mathrm{b}}$ \\
\hline $\begin{array}{l}\text { Histological analysis at baseline }[\mathrm{n}(\%)] \\
\text { PAS stain }(+) \\
\text { PAS stain }(-)\end{array}$ & $\begin{array}{l}10(100) \\
0(0)\end{array}$ & $\begin{array}{l}10(100) \\
0(0)\end{array}$ \\
\hline Significance between groups & \multicolumn{2}{|c|}{$p>0.05$} \\
\hline $\begin{array}{l}\text { Histological analysis at } 16-\mathrm{w} \text { follow-up [n (\%)] } \\
\text { PAS stain }(+) \text { culture }(+) \\
\text { PAS stain }(-) \text { culture }(-)\end{array}$ & $\begin{array}{l}8(80) \\
2(20)\end{array}$ & $\begin{array}{l}10(100) \\
0(0)\end{array}$ \\
\hline Significance between groups & \multicolumn{2}{|c|}{$p>0.05$} \\
\hline $\begin{array}{l}\text { Histological analysis at } 40-\mathrm{w} \text { follow-up [n (\%)] } \\
\text { PAS stain }(+) \text { culture }(+) \\
\text { PAS stain }(-) \text { culture }(-)\end{array}$ & $\begin{array}{l}3(30)^{\mathbf{a}, \mathbf{b}} \\
7(70)^{\mathbf{a}, \mathbf{b}}\end{array}$ & $\begin{array}{l}4(40)^{\mathbf{a}, \mathbf{b}} \\
6(60)^{\mathbf{a}, \mathbf{b}}\end{array}$ \\
\hline Significance between groups & \multicolumn{2}{|c|}{$p>0.05$} \\
\hline
\end{tabular}

(a) Intragroup significance between the baseline and follow-up values

(b) Intragroup significance between the 16-week follow-up and 40-week follow-up values 
Table 3.- Cure rates at 16 and 40 -week follow-ups. $((\mathrm{n}=$ number of patients; $(\%)=$ percentage of patients)

\begin{tabular}{|l|c|c|c|c|}
\cline { 2 - 5 } \multicolumn{1}{c|}{} & \multicolumn{2}{c|}{ MB/PDT } & \multicolumn{2}{c|}{ MAL/PDT } \\
\cline { 2 - 5 } \multicolumn{1}{c|}{} & $\begin{array}{c}16 \text { weeks } \\
\mathrm{n}(\%)\end{array}$ & $\begin{array}{c}40 \text { weeks } \\
\mathrm{n}(\%)\end{array}$ & $\begin{array}{c}16 \text { weeks } \\
\mathrm{n}(\%)\end{array}$ & $\begin{array}{c}40 \text { weeks } \\
\mathrm{n}(\%)\end{array}$ \\
\hline Mycological cure & $2(20)$ & $7(70)$ & $0(0)$ & $6(60)$ \\
\hline Clinical cure & $2(20)$ & $7(70)$ & $0(0)$ & $4(40)$ \\
\hline Complete cure & $2(20)$ & $7(70)$ & $0(0)$ & $4(40)$ \\
\hline Treatment success & $0(0)$ & $0(0)$ & $0(0)$ & $1(10)$ \\
\hline Clinical improvement & $0(0)$ & $0(0)$ & $0(0)$ & $1(10)$ \\
\hline
\end{tabular}




\section{Figure captions}

Figure 1.- Participant flow diagram for clinical trial.

Figure 2.- Histological analysis of a nail of one patient through PAS stain: (A) before the study, where the presence of fungal elements can be seen; (B) after the study, without presence of fungal elements.

Figure 3.- Clinical evolution of two toenail treated for onychomycosis with MB/PDT (A,B) and MAL/PDT (C,D), respectively: (A) before treatment (30\% of nail involvement), (B) at 24 week after MB/PDT (40-week follow-up) (2\% of nail involvement) (C) before treatment ( $40 \%$ of nail involvement) and (D) at 24 week after MAL/PDT (40-week follow-up) (5\% of nail involvement). 
Assessed for eligibility $(n=36)$

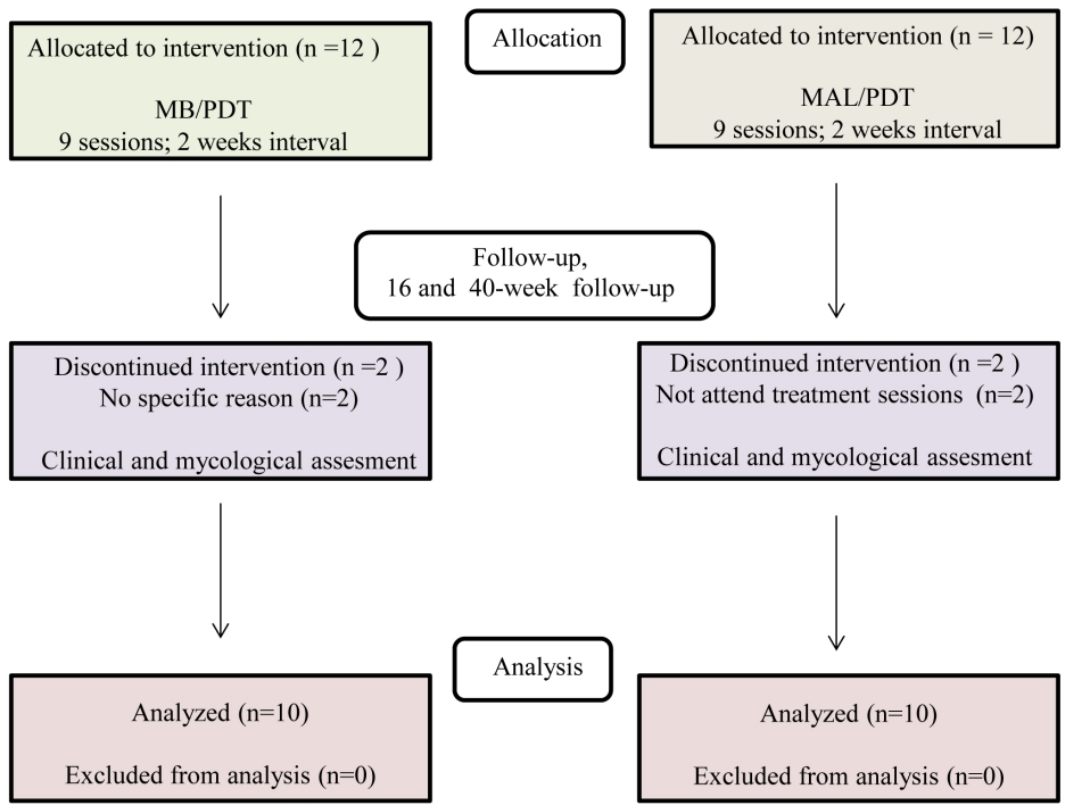

Figure 1

Figure 1.- Participant flow diagram for clinical trial. 

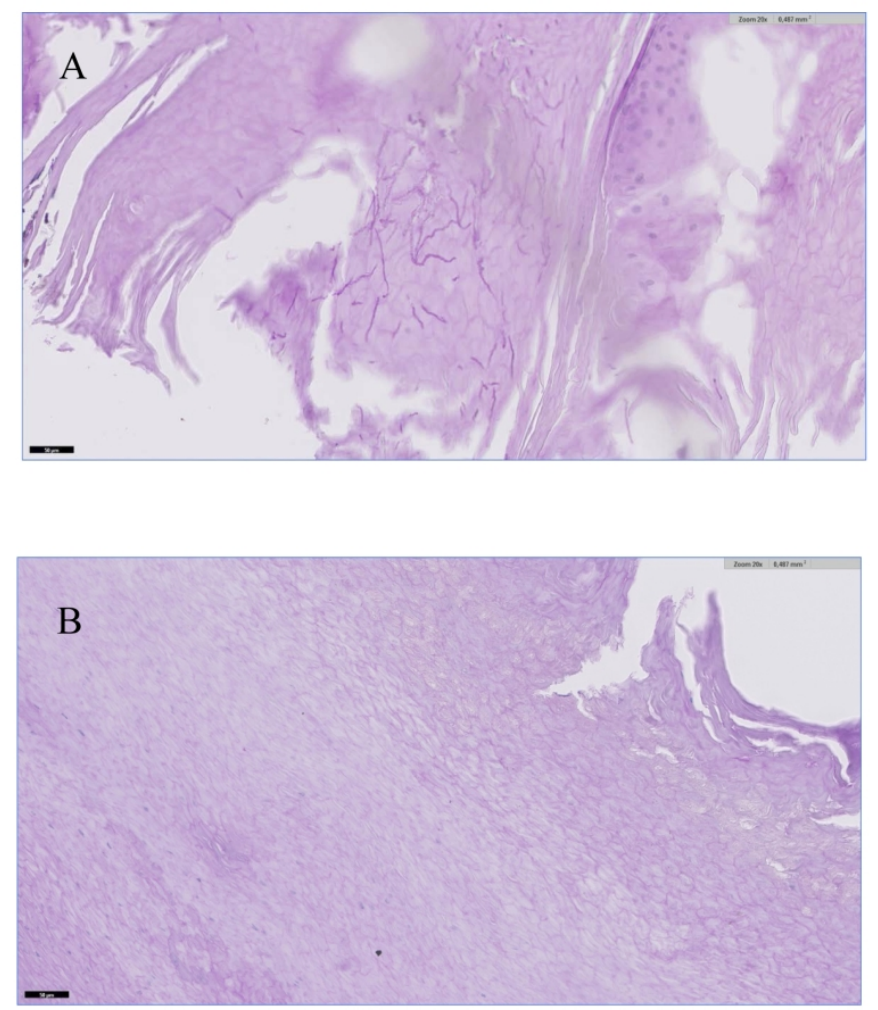

Figure 2

Figure 2.- Histological analysis of a nail of one patient through PAS stain: (A) before the study, where the presence of fungal elements can be seen; (B) after the study, without presence of fungal elements. 

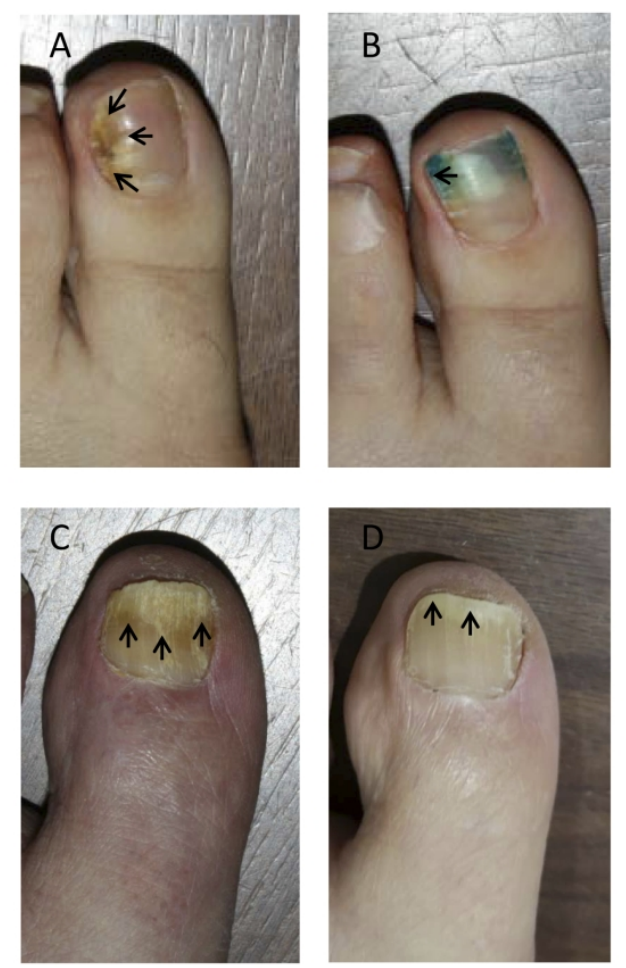

Figure 2

Figure 3.- Clinical evolution of two toenail treated for onychomycosis with MB/PDT ( $A, B)$ and MAL/PDT (C,D), respectively: $(A)$ before treatment (30\% of nail involvement), (B) at 24 week after MB/PDT (40-week followup) ( $2 \%$ of nail involvement) (C) before treatment ( $40 \%$ of nail involvement) and (D) at 24 week after MAL/PDT (40-week follow-up) ( $5 \%$ of nail involvement). 